\title{
Quantificação de fósforo por Mehlich 1, Mehlich 3 e Resina Trocadora de Ânions em solos com diferentes teores de argila
}

\author{
Gilmar Luiz Mumbach ${ }^{1 *}$, Daniela Aparecida de Oliveira ${ }^{1}$, Maria Izabel Warmling ${ }^{1,}$ Luciano Colpo Gatiboni $^{1}$
}

\author{
$10.1590 / 0034-737 X 201865060010$
}

\section{RESUMO}

Os extratores de fósforo (P) Mehlich 1 (M-1), Mehlich 3 (M-3) e Resina Trocadora de Ânions (RTA) apresentam sensibilidade ao conteúdo de argila do solo. Ainda, o uso de equações para conversão do P disponível extraído por M3 em valores equivalentes de M-1 pode não ser adequado para um conjunto de solos contrastantes. O objetivo do trabalho é avaliar a influência do conteúdo de argila na extração de P por M-1, M-3 e RTA, bem como avaliar a efetividade de utilização de equações para conversão dos teores por M-3 em valores equivalentes de M-1. Foram selecionadas 301 amostras de solo no laboratório de rotina do CAV/UDESC, distribuídos em quatro classes de argila: 0-20, 21-40, 41-60 e > 60\% de argila. As amostras foram preparadas e foi extraído o P disponível por M-1, M-3 e RTA. Os extratores M-3 e RTA extraíram quantidades de P 12 e $11 \%$ superiores ao M-1, respectivamente, M-1 extraiu menos P que M-3 e RTA em solos de textura arenosa e extraiu mais P que os demais extratores em solos de textura argilosa. A equação sugerida pela CQFS - RS/SC (2016) para conversão dos teores de M-3 para equivalentes em M-1 subestimou os valores de $\mathrm{P}$ em até $24 \%$. Com isso, alternativamente, se propõe a utilização da equação P-M1 ${ }_{\text {eq }}\left(\mathrm{mg} \mathrm{dm}{ }^{-3}\right)=(\mathrm{P}-\mathrm{M} 3$ / (1,38-0,01*Arg)). Os métodos avaliados apresentam sensibilidade ao teor de argila do solo, devendo ser realizada a classificação da disponibilidade $\mathrm{P}$ por faixas de textura.

Palavras-chave: extratores de P; análise do solo; fosfato solúvel; Santa Catarina.

\section{ABSTRACT}

\section{Phosphorus extraction by Mehlich 1, Mehlich 3, and Anion Exchange Resin in soils with different clay contents}

Phosphorus (P) extractors Mehlich 1 (M-1), Mehlich 3 (M-3), and Anion Exchange Resin (RTA) show sensitivity to soil clay content. In addition, the use of equations for conversion of available $\mathrm{P}$ extracted by M-3 into equivalent values of M-1 may not be adequate for a set of contrasting soils. The objective of this work was to evaluate the influence of clay content on the extraction of P by M-1, M-3, and RTA, as well as to evaluate the effectiveness of using the equation proposed by Local Soil Fertility Committee to convert M-3 contents into equivalent M-1 values. We selected 301 soil samples from the soil test laboratory at Santa Catarina State University, distributed into four classes of clay: 0-20, 2140, 41-60, and > 60\% clay. The samples were prepared, and the available P was extracted by M-1, M-3, and RTA. M-3 and RTA extracted 12 and $11 \%$ more P than M-1, but M-1 extracted less P than M-3 and RTA in sandy soils and extracted more $\mathrm{P}$ than the extractors in clayey soils. The equation suggested by Local Soil Fertility Committee for converting M3 values into equivalents in $\mathrm{M}-1$ underestimated $\mathrm{P}$ values by up to $24 \%$; therefore, we suggest the use of the equation $\mathrm{P}-\mathrm{M} 1_{\text {eq }}\left(\mathrm{mg} \mathrm{dm}^{-3}\right)=(\mathrm{P}-\mathrm{M} 3 /(1.38-0.01 * \mathrm{Arg}))$. The evaluated methods present sensitivity to soil clay content, and the classification of $\mathrm{P}$ availability should be performed by texture bands.

Keywords: extractors of P; soil analysis; soluble phosphate; Santa Catarina.

Submetido em 26/03/2018 e aprovado em 19/11/2018.

${ }^{1}$ Universidade do Estado de Santa Catarina, Centro de Ciências Agroveterinárias, Departamento de Ciência do Solo e Recursos Naturais, Lages, Santa Catarina, Brasil. gilmarmumbach@hotmail.com; danioliveira7@yahoo.com.br; bel_warmling@hotmail.com; lgatiboni@gmail.com

"Autor para correspondência: gilmarmumbach@ hotmail.com 


\section{INTRODUÇÃO}

O fósforo $(\mathrm{P})$ é um macronutriente essencial às plantas e sua disponibilidade é influenciada pela interação às partículas sólidas do solo (Gatiboni et al., 2008; Chien et al., 2014). No solo, o P é encontrado ligado a frações orgânicas e inorgânicas, com elevada afinidade à fração argila, realizando ligações de alta energia, especialmente a óxidos de ferro e alumínio (Eriksson et al., 2015; Fink et al., 2016).

Existem diversos métodos utilizados para extração do "P disponível". Nos estados do Rio Grande do Sul (RS) e Santa Catarina (SC) utiliza-se o extrator Mehlich 1 (M-1) como o método oficial. Esse método foi calibrado para as principais espécies agrícolas cultivadas na região e apresenta como vantagem sua facilidade de execução (Oliveira, 2010). Contudo, extrai, preferencialmente, as formas de P ligadas a cálcio (Mehlich, 1978), superestimando a disponibilidade de $\mathrm{P}$ em solos recentemente adubados com fosfatos naturais (Bortolon et al., 2011), perdendo a sua capacidade de extração em solos mais argilosos (Oliveira, 2010; Freitas et al., 2013). Com isso, fez-se necessário a criação de grupos de solos com teores de argila semelhantes para a interpretação do teor de $\mathrm{P}$ de maneira relativa em cada intervalo de textura (CQFS, RS/SC, 2016). Mesmo com essas limitações, o M-1 tem sido usado como método oficial no RS e SC desde 1968, tendo boa capacidade de predição da disponibilidade de $\mathrm{P}$, exceto em solos com aplicação recente de fosfatos naturais.

O método da resina trocadora de ânions (RTA) era recomendado até meados de 2016 pela Comissão de Química e Fertilidade do Solo dos estados do RS e SC (CQFS - RS/SC) para quantificar a disponibilidade de $\mathrm{P}$ em solos que receberam adição de fosfatos naturais mas, por dificuldades operacionais dos laboratórios de rotina, o serviço era raramente oferecido . Em substituição, passou a ser recomendado como alternativa o método Mehlich 3 (M3). O M-3 minimiza a influência dos fosfatos naturais no resultado analítico (Oliveira et al., 2015), além de ter a vantagem de ser um método multielementar, podendo-se determinar, além de $\mathrm{P}$, outros nutrientes como potássio (K), Cálcio (Ca), magnésio (Mg), manganês (Mn), zinco ( $\mathrm{Zn})$ e cobre (Cu) (Mehlich, 1984).

A adoção de uma nova metodologia para estimar a disponibilidade para as plantas deve estar respaldada numa ampla calibração de campo, onde os teores extraídos são correlacionados com o desempenho das plantas. Para o M-3 ainda há um pequeno conjunto de dados no RS e SC para suportar uma calibração, enquanto para o M-1 a calibração tem sido ajustada através de experimentos realizados por mais de 50 anos. Assim, enquanto o conjunto de dados é incipiente, a última edição do Manual de Calagem e Adubação para os estados do RS e SC
(CQFS - RS/SC - 2016), adotou a estratégia de transformar os teores de P extraídos por M-3 em "equivalentes a M-1" e, posteriormente utilizar as tabelas de interpretação já calibradas para M-1. Para isto, o manual sugere a utilização de uma equação (P M-1 = P M-3/ (2 - (0,02*argila)). Essa equação foi baseada nos trabalhos de Bortolon \& Gianello $(2008,2012)$ com solos da região, mostrando que o M-3 extrai mais P que o M-1 em solos arenosos e menos P que o M-1 em solos argilosos, ou seja, o M-3 tem alta sensibilidade ao teor de argila do solo.

O método M-3 apresenta grande potencial para utilização dos estados do RS e SC, podendo futuramente vir a substituir o M-1. Para isso, necessita-se em longo prazo promover redes de experimentos de calibração de campo usando o método e em curto prazo, precisa-se avaliar se a equação de transformação de M-3 em equivalentes M-1 proposta pela CQFS - RS/SC (2016) é adequada para um conjunto amplo de solos dos estados de abrangência CQFS - RS/SC. A hipótese do estudo é que a capacidade de extração dos métodos M-1, M-3 e RTA varia de acordo com os teores de argila do solo e os objetivos foram correlacionar os teores extraídos pelos métodos M-3 e RTA com o método padrão M-1 em 301 amostras de solos do Estado de Santa Catarina com diferentes teores de argila e avaliar a exatidão da equação proposta pela CQFSRS/SC (2016) para transformação dos teores de P extraídos por M-3 em equivalentes a M-1.

\section{MATERIAL E MÉTODOS}

O estudo foi desenvolvido no laboratório de química e fertilidade do solo do Centro de Ciências Agroveterinárias, na Universidade do Estado de Santa Catarina, em Lages, SC. Para realização do trabalho foram selecionadas 301 amostras de solos de Santa Catarina, visando abranger uma ampla variabilidade de teores de argila. Assim, compreenderam amostras com teor de argila < 20\% (n: 41); entre 21 e $40 \%$ (n: 116); 41 e $60 \%$ (n: 91); e $>61 \%$ (n: 53). As amostras foram secas em estufa com circulação forçada de ar à $65^{\circ} \mathrm{C}$, moídas em moinho de martelo e tamisadas em peneira de $2 \mathrm{~mm}$. Após o preparo as amostras foram submetidas à caracterização química (Tedesco et al., 1995) e granulométrica (Embrapa, 1997) (Tabela 1).

A disponibilidade de $\mathrm{P}$ foi avaliada por três métodos de extração: Mehlich 1 (M-1), Mehlich 3 (M-3) e Resina Trocadora de Ânions em Lâminas (RTA). O extrator M-1 foi preparado com a mistura de dois ácidos diluídos $(0,05$ mol L-1 de $\mathrm{HCl}$ e $0,0125 \mathrm{~mol} \mathrm{~L}^{-1} \mathrm{de}_{2} \mathrm{SO}_{4}$ ). O processo de extração consistiu da adição da solução extratora ao solo (relação solo: solução de 1:10), com posterior agitação de 5 minutos, e seguida por decantação de 16 horas (Tedesco et al.,1995). Após o período de decantação retirou-se uma 


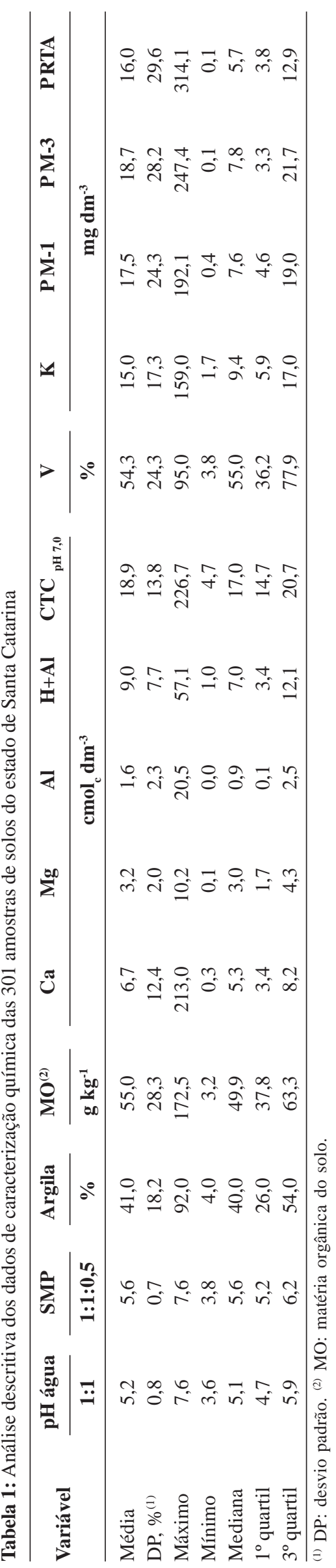

alíquota do extrato para determinação da concentração de P. A solução extratora M-3 foi preparada de acordo com Mehlich (1984), com os seguintes reagentes e concentrações: $\mathrm{CH}_{3} \mathrm{COOH}\left(0,2 \mathrm{~mol} \mathrm{~L}^{-1}\right) ; \mathrm{NH}_{4} \mathrm{NO}_{3}(0,25 \mathrm{~mol} \mathrm{~L}-$ 1); $\mathrm{NH}_{4} \mathrm{~F}\left(0,015 \mathrm{~mol} \mathrm{~L}^{-1}\right) ; \mathrm{HNO}_{3}\left(0,013 \mathrm{~mol} \mathrm{~L}^{-1}\right) \operatorname{eEDTA}(0,001$ $\left.\mathrm{mol} \mathrm{L}^{-1}\right)$. O processo de extração foi idêntico ao realizado para M-1. Para extração com RTA em lâminas foi utilizada a metodologia descrita por Miola (1995) e adaptações de Gatiboni et al. (2005), usando uma relação solo: resina de $1,0 \mathrm{~g}: 2,5 \mathrm{~cm}^{2}$. A determinação da concentração de P para os três extratores foi realizada pelo método da quantificação do complexo fosfomolibdato em espectrofotômetro de absorção molecular, com leitura em comprimento de onda de $882 \mathrm{~nm}$, seguindo metodologia descrita por Murphy \& Riley (1962).

A partir dos teores de $\mathrm{P}$ obtidos pelo extrator $\mathrm{M}-3$, calcularam-se os valores de "M-1 equivalente" (P M-1 $\mathrm{CQFS}$ ) através da equação proposta pela CQFS - RS/SC (2016) $[\mathrm{P} \mathrm{M}-1$ = P M-3/ 2 - (0,02*argila)], proposta no Manual de Calagem e Adubação para os estados do RS e SC (CQFS - RS/SC, 2016). Os cálculos foram realizados para 301 amostras conjuntamente e separadamente para os quatro grupos de argila. Adicionalmente, foi ajustada uma nova equação para cálculo do "P M-1 equivalente" $\left(\mathrm{P}-\mathrm{M} 1_{\text {eq }}\right)$, a qual foi gerada através da correlação entre os valores centrais da amplitude das classes de argila e o coeficiente angular das equações que descreveram os valores extraídos por M-1 e M-3, dentro das quatro classes de textura.

Os dados coletados foram submetidos à análise de normalidade pelo teste de Shapiro-Wilk. Posteriormente, com os resultados obtidos, foi realizada a correlação de Pearson e as equações foram ajustadas para modelos lineares. A análise estatística foi realizada com auxílio do programa Sisvar 5.6 (Ferreira, 2014) e a confecção dos gráficos com o programa SigmaPlot 12.5 .

\section{RESULTADOS E DISCUSSÃO}

Os valores de matéria orgânica (MO) apresentaram correlação significativa com os valores de $\mathrm{P}$ extraídos por M-1, M-3 e RTA (Figura 1A). O aumento dos teores de matéria orgânica foi acompanhado pelo aumento dos teores de $\mathrm{P}$ extraídos pelos três métodos, atribuindo-se este resultado à capacidade do carbono de interagir com óxidos de Fe e Al presentes no solo e reduzir a sorção de $\mathrm{P}$ a esses compostos (Chien et al., 2014; Eriksson et al., 2015; Fink et al., 2016). Além disso, solos com maiores teores de MO tendem a contribuir com maiores valores de $\mathrm{P}$ à planta, apesar de os principais métodos de rotina não quantificar grande parte do $\mathrm{P}$ orgânico (Steffens $e t$ al., 2010). Contudo, a correlação entre MO e disponibilidade de $\mathrm{P}$ por vezes é baixa, como observado no presen- 
te estudo, em função de que a contribuição da MO na disponibilidade de $\mathrm{P}$ é dependente da relação $\mathrm{C}: \mathrm{P}$ do material orgânico, a qual é bastante variável (Camargo et al., 2013).

Os aumentos dos teores de argila foram acompanhados pela redução na disponibilidade de P extraído por M1, M-3 e RTA (Figura 1B), indicando que todos os métodos foram sensíveis ao poder tampão do solo. Solos mais argilosos, apesar do maior conteúdo total de P, apresentam menor quantidade facilmente extraível do nutriente, em função da maior capacidade sortiva destes solos, devido à presença de maiores concentrações de óxidos de Fe e Al (Oliveira Júnior et al., 2011; Eriksson et al., 2015). Além disso, a capacidade extratora dos métodos, especialmente de M-1, é reduzida em solos com elevados teores de argila em função da exaustão do extrator e da readsorção de $\mathrm{P}$ durante o tempo de repouso (Steiner et al., 2012; Freitas et al., 2013).

Os extratores M-3 e RTA apresentaram elevada correlação com os valores de P obtidos por M-1 para as 301 amostras de solo analisadas (Figura 2). Contudo, os métodos M-3 e RTA extraíram 12\% e $11 \%$ de P a mais que M1 , respectivamente. Resultados similares já foram apresentados em outros estudos, com maiores valores extraídos por M-3 e RTA em comparação à M-1 (Bortolon \& Gianello, 2008; Bortolon et al., 2009; Bortolon et al., 2011; Schlindwein et al., 2011; Steiner et al., 2012). Essa diferença entre os métodos pode estar relacionada aos seus diferentes mecanismos de extração. Os maiores valores de $\mathrm{P}$ extraídos por M-3 podem ser relacionados ao fato de este método retirar preferencialmente $\mathrm{P}$ ligado a Fe e Al, formas predominantes nos solos do Sul do Brasil, enquanto
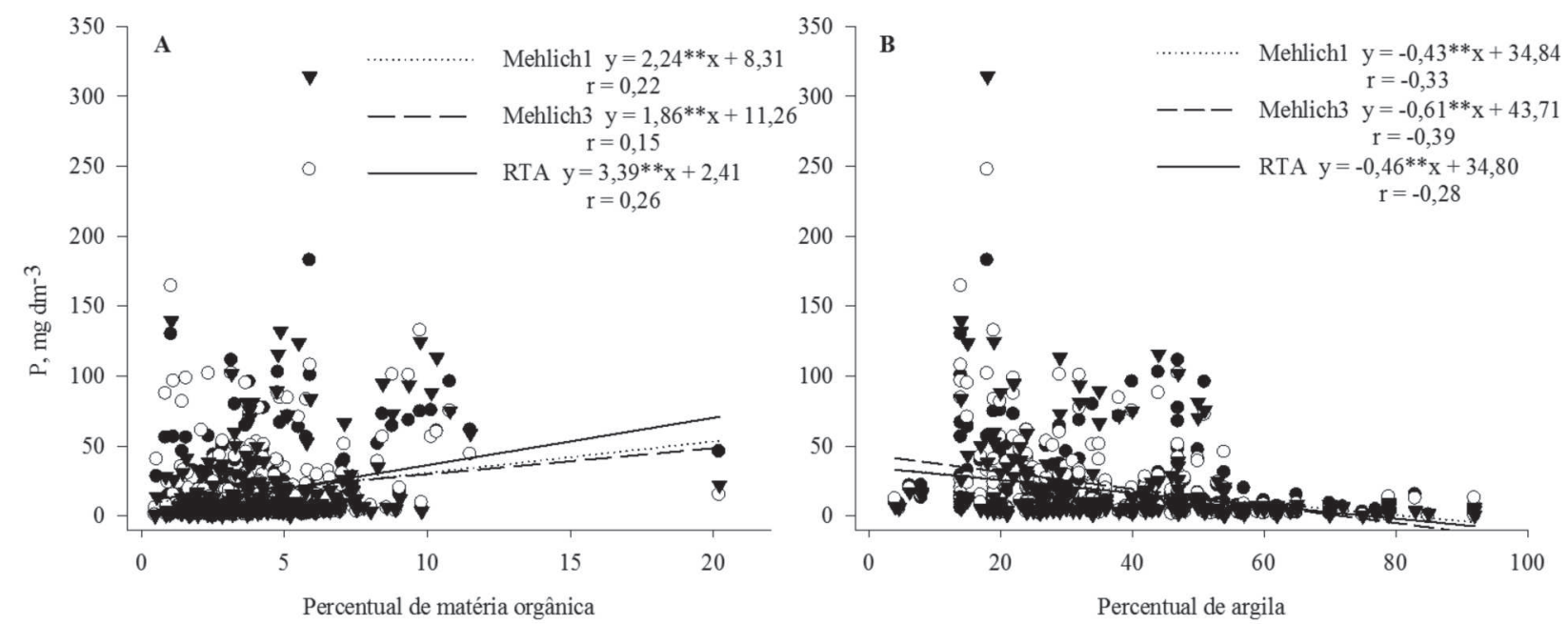

Figura 1: Influência do conteúdo de matéria orgânico (A) e dos teores de argila do solo (B) sobre o P extraído pelos métodos Mehlich 1 (o), Mehlich $3(\bullet)$ e RTA ( $\mathbf{v})$ para 301 solos catarinenses.

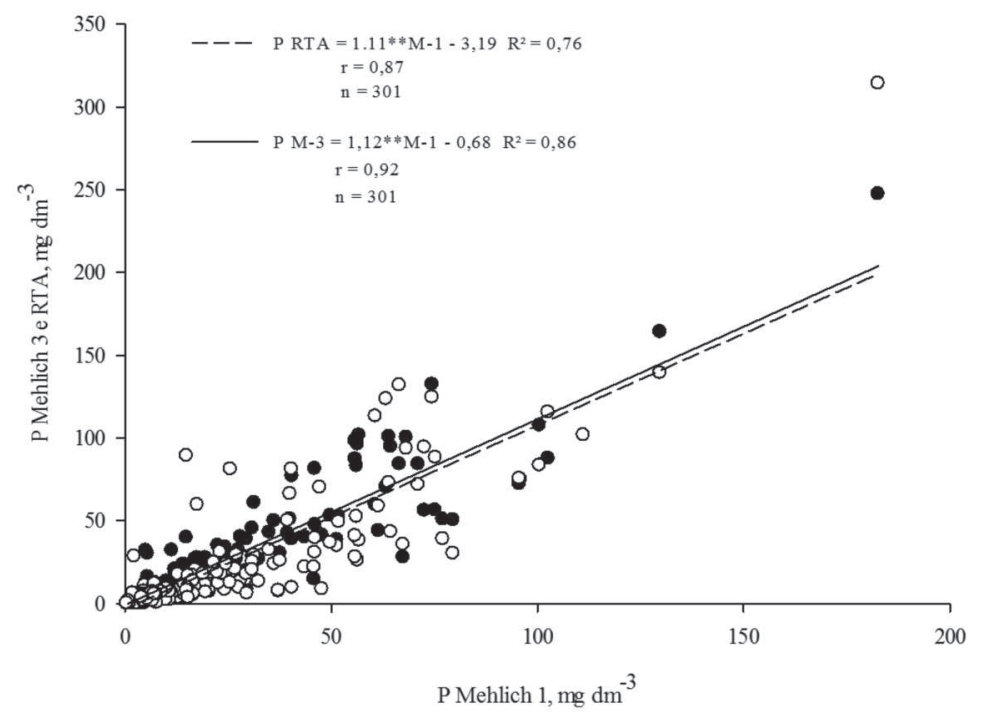

Figura 2: P disponível extraído por Mehlich 1 e pelos extratores Mehlich 3 (o) e RTA (•) em 301 amostras de solos do estado de Santa Catarina. 
o método M-1 extrai preferencialmente as formas de $\mathrm{P}$ ligado ao Ca (Bortolon et al., 2009). Além disso, o extrator M-3 pela presença de EDTA, pode extrair algumas formas orgânicas de P (Gatiboni et al., 2005). Os menores valores de $\mathrm{P}$ extraídos por M-1 podem ser atribuídos à alta capacidade de troca de cátions e mineralogia dos solos utilizados, havendo uma neutralização dos ácidos diluídos (Bortolon et al., 2011) e exaustação da capacidade de extração, principalmente nos solos mais argilosos e intemperizados (Santos et al., 2008). Adicionalmente, na extração com M-1 há possibilidade de haver readsorção de parte do P extraído durante as 16 horas de decantação, fenômeno este menos expressivo aos demais extratores (Steiner et al., 2012). As maiores quantidades de P extraídas pelo método da RTA em comparação a M-1 podem ocorrer em função do longo tempo de agitação e saturação da RTA com ânion bicarbonato, o qual extrai P adsorvido as superfícies dos colóides até um equilíbrio eletroquímico entre o solo e a superfície da resina (Bortolon et al., 2009; Bortolon et al., 2011).

O comportamento entre os três métodos de extração de $\mathrm{P}$ variou de acordo com o conteúdo de argila do solo (Figura 3). Considerando os solos com teores até $20 \%$ de

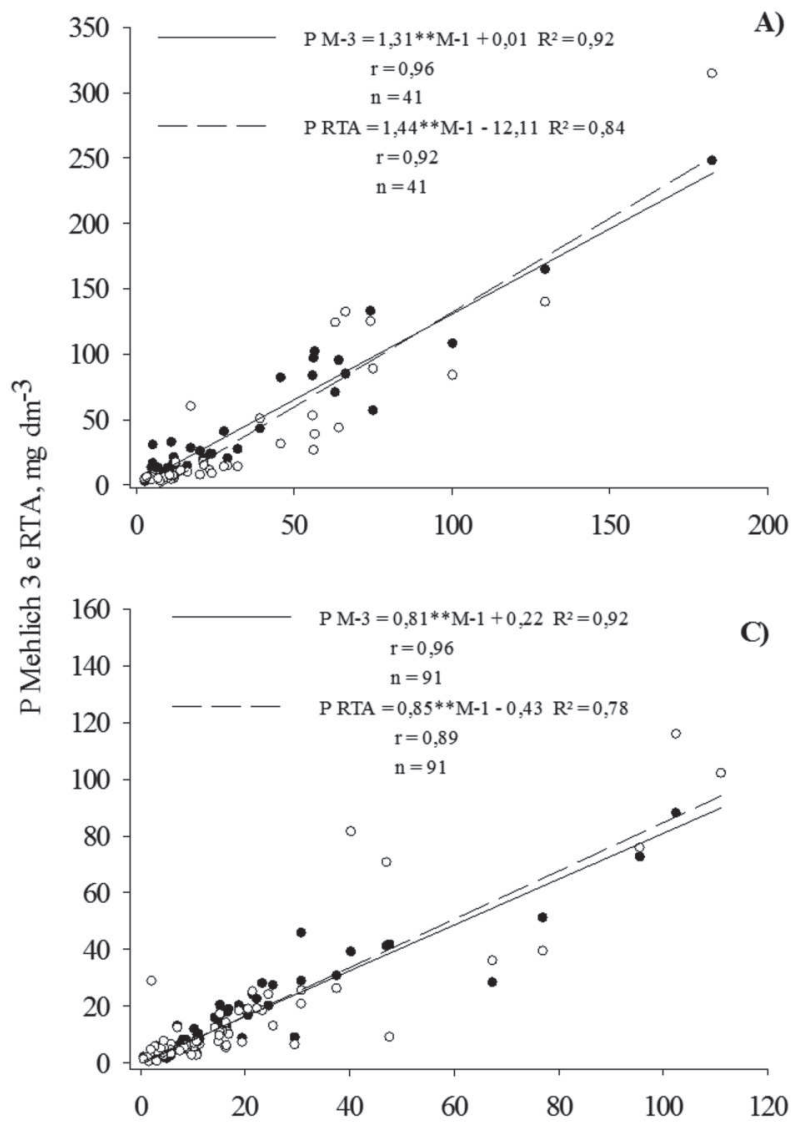

argila, os métodos M-3 e RTA extraíram 31 e 44\% mais P do que o método M-1 (Figura 3A). Para os solos com teores de argila variando entre 21 e $40 \%$ os valores extraídos por M-1 e M-3 foram muito próximos, com uma ligeira maior extração por M-3 (2\%), enquanto o extrator RTA apresentou valores de $\mathrm{P}$ aproximadamente $9 \%$ inferiores que M-1(Figura 3B). Para solos com 41 a $60 \%$ de argila passa a ocorrer um comportamento inverso, onde M-1 extraiu cerca de $19 \%$ a mais de P que M-3 e $11 \%$ superiores a RTA (Figura 3C). Para os solos com mais de $60 \%$ de argila os teores de P extraído por M-1 foram cerca de 19\% superiores a M-3 e cerca de $30 \%$ superiores a RTA (Figura 3D). O comportamento diferenciado entre M-1 e M-3 em função da classe de argila também foi observado por Bortolon \& Gianello (2008) utilizando amostras do estado do Rio Grande do Sul, com maiores valores extraídos por M-3 em solos com até $40 \%$ de argila, enquanto que para solos com mais de $40 \%$ de argila o método M-1 extrai valores superiores de P. No entanto, o comportamento entre M-1 e RTA diferiu ao observado em outros estudos (Schlindwein \& Gianello, 2008; Schlindwein et al., 2011; Freitas et al., 2013), onde, de maneira geral, M-1 extrai maior quantidade de $\mathrm{P}$ em solos arenosos e menor em
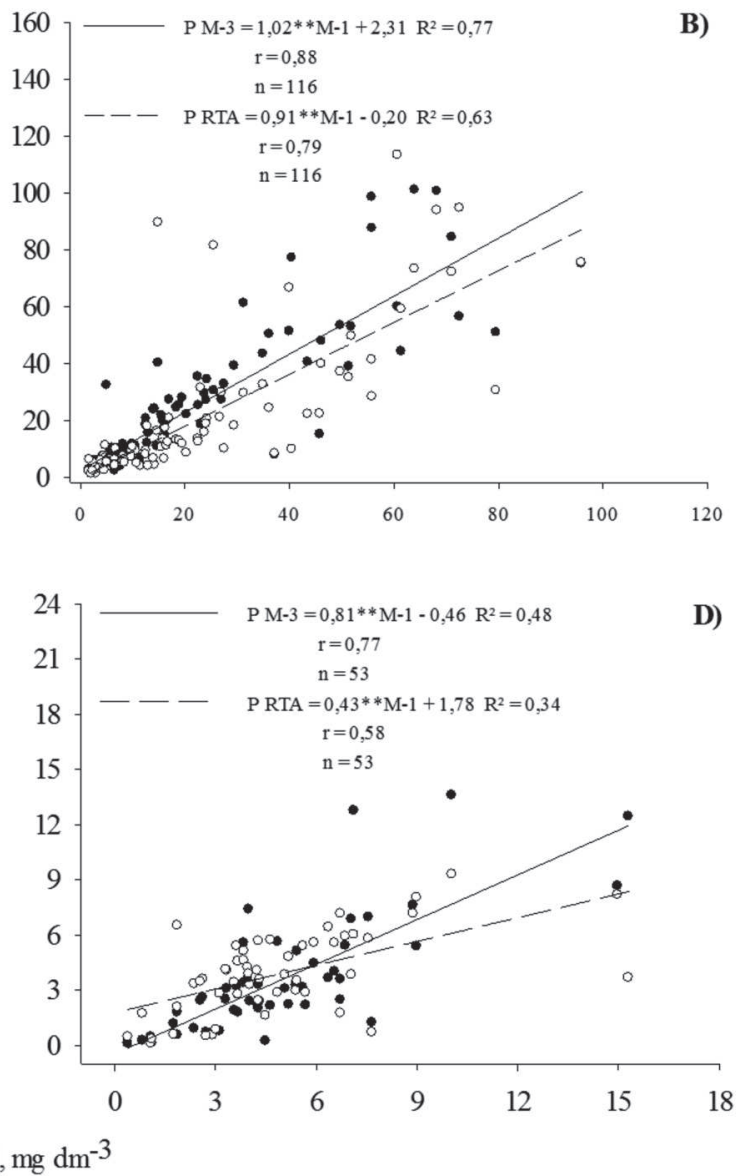

Figura 3: P disponível extraído por Mehlich 1 e pelos extratores Mehlich 3 (o) e RTA (•) em 301 amostras de solos do estado de Santa Catarina, agrupadas de acordo com classes texturais, sendo: (A) solos de 0 a $20 \%$ de argila, (B) de 21 a $40 \%$, (C) de 41 a $60 \%$ e (D) $>60 \%$ de argila.

Rev. Ceres, Viçosa, v. 65, n.6, p. 546-554, nov/dez, 2018 


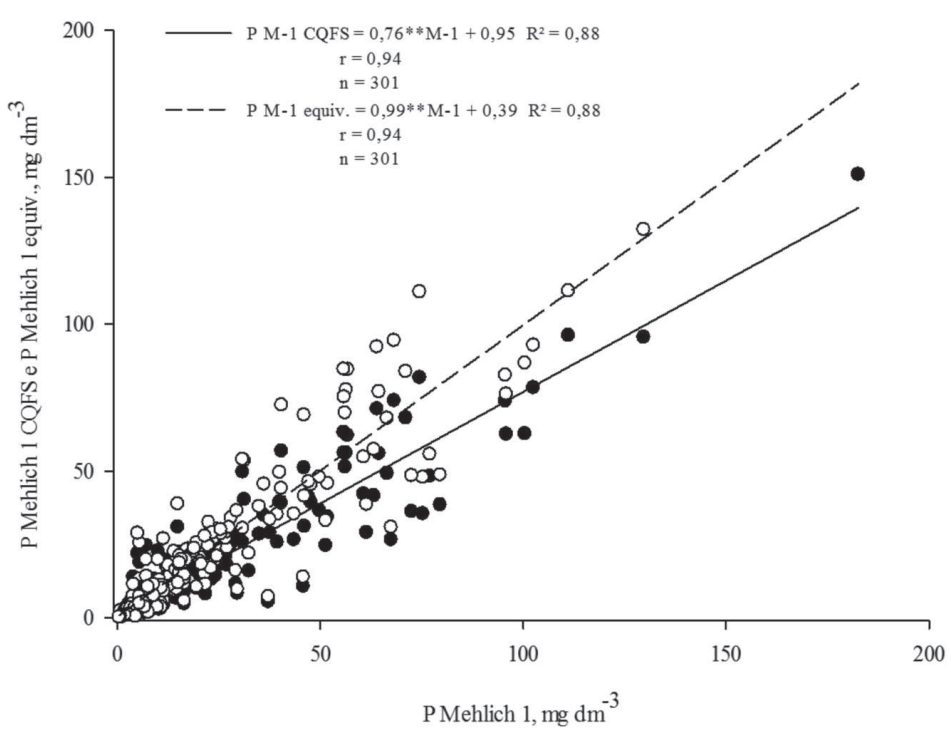

Figura 4: P extraído por Mehlich 1 e valores de equivalentes a Mehlich 1 calculados a partir dos teores extraídos por Mehlich 3, usando a equação proposta pela CQFS $-\mathrm{RS} / \mathrm{SC}\left(\mathrm{P}-\mathrm{M} 1_{\mathrm{eq}}=(\mathrm{P}-\mathrm{M} 3 /(2-(0,02 * \arg ))(\mathrm{o})\right.$ e usando a equação sugerida no presente estudo $\left(\mathrm{P}-\mathrm{M} 1_{\mathrm{eq}}=(\mathrm{P}-\mathrm{M} 3 /(1,38-(0,01 * \arg ))(\bullet)\right.$.
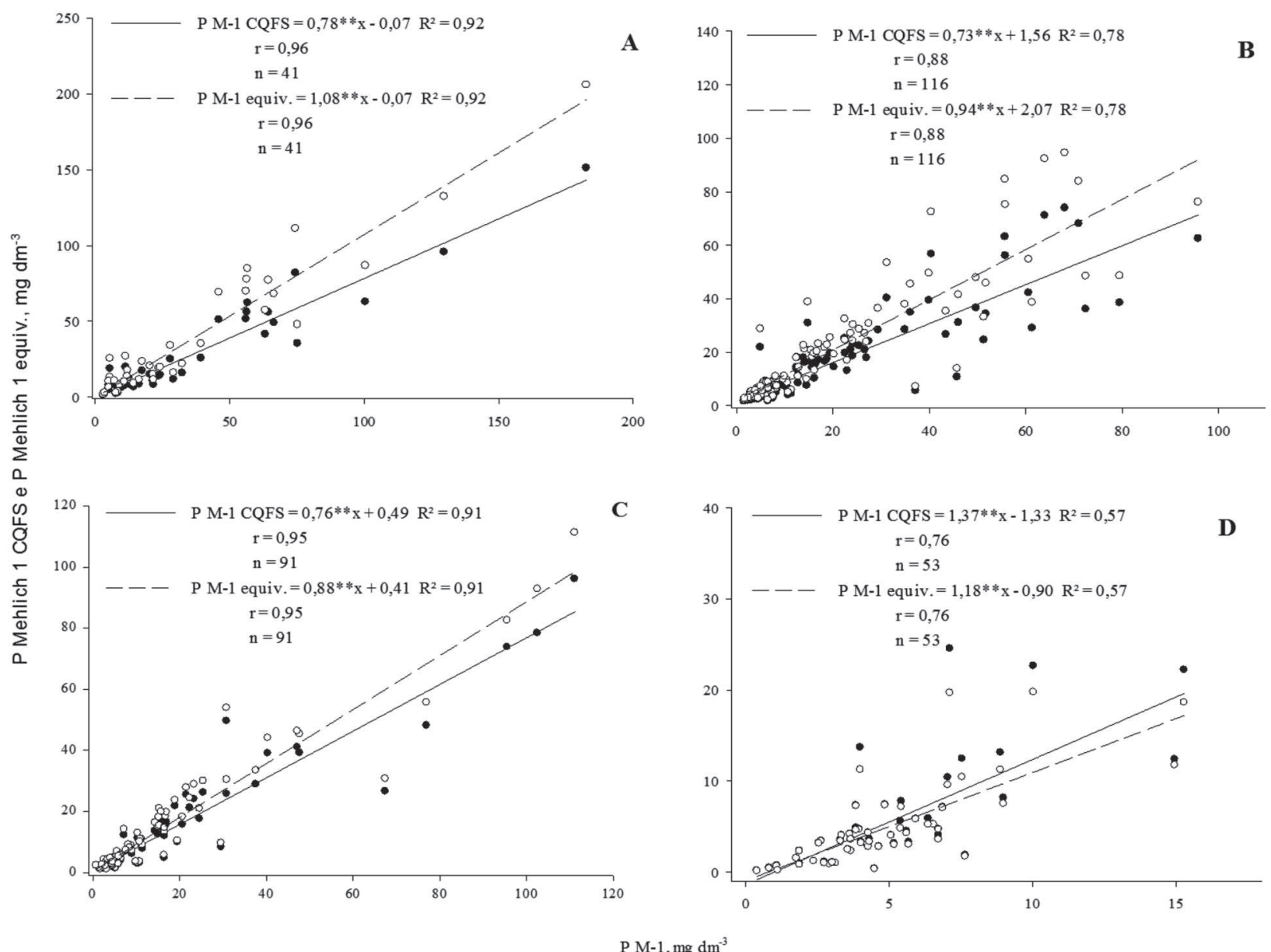

Figura 5: $\mathrm{P}$ extraído por Mehlich 1 e valores de equivalentes a Mehlich 1 calculados a partir dos teores extraídos por Mehlich 3, usando a equação proposta pela CQFS - RS/SC $\left(\mathrm{P}-\mathrm{M} 1_{\mathrm{eq}}=(\mathrm{P}-\mathrm{M} 3 /(2-(0,02 * \mathrm{arg}))(\mathrm{O})\right.$ e usando a equação sugerida no presente estudo $\left(\mathrm{P}-\mathrm{M} 1_{\mathrm{eq}}=(\mathrm{P}-\mathrm{M} 3 /(1,38-(0,01 * \mathrm{arg}))(\bullet)\right.$. As amostras foram agrupadas de acordo com classes texturais, sendo: (A) solos de 0 a $20 \%$ de argila, (B) de 21 a 40\%, (C) de 41 a $60 \%$ e (D) $>60 \%$ de argila. 
solos argilosos, em comparação a RTA. De acordo com Bortolon et al. (2009), em solos com altos teores de argila, maiores valores de P são extraídos por RTA, em comparação com M-1, o que pode ser atribuído à ocorrência de readsorção de P no método de M-1, sendo as diferenças dos dois métodos mais pronunciadas em solos com alta disponibilidade desse elemento. Menores teores de P extraídos por RTA e por M-3, em comparação a M-1, em solos argilosos, podem indicar sensibilidade dos métodos ao conteúdo de argila presente nos solos da região Sul do Brasil.

Os valores de P estimados através da equação proposta pela CQFS-RS/SC (2016) (P M-1 CQFS), utilizando os valores determinados por M-3, foram cerca de $24 \%$ inferiores aos valores reais determinados por M-1 (Figura 4). Esse mesmo comportamento foi observado com o agrupamento dos solos pelos diferentes teores de argila (Figura 5). Os valores determinados de M-1 foram superiores aos calculados pela equação nos solos com menos de $20 \%$ de argila, com 21 a $40 \%$ e com 41 a $60 \%$ de argila (Figura 5A, 5B, 5C). Para solos com mais de 60\% de argila ocorreu um comportamento contrário, onde os valores de $\mathrm{P}$ calculados pela equação foram cerca de $30 \%$ superiores aos determinados pela extração com M1. Diante dessa variação, a utilização de uma equação para converter os valores determinados pela extração com M- 3 em valores equivalentes a M-1 pode apresentar certas limitações. Essa variação pode ocorrer devido a variabilidade dos solos presentes nos estados do RS e $\mathrm{SC}$, em relação ao material de origem, clima e uso e manejo do solo, dentre outros fatores, levando a diferentes características em termos de tamponamento e disponibilidade de nutrientes (Almeida et al., 1999). Desta forma, com os resultados encontrados para os valores de $\mathrm{P}$ determinados pelos extratores M-1 e M-3, no presente trabalho sugere-se uma nova equação $\left[\mathrm{P}-\mathrm{M} 1_{\mathrm{eq}}=(\mathrm{P}-\mathrm{M} 3 /\right.$ $(1,38-(0,01 * a r g)]$, a qual apresenta melhor estimativa entre os valores determinados e calculados para M-1 (Figura 5), para esse grupo de solos. Alternativamente, podese converter os valores de M-3 para equivalentes em M1 segundo a classe de textura, conforme a seguir: solos com argila <20\%: P-M1 eq $=\mathrm{P}-\mathrm{M} 3 * 1,3$; solos com argila

Tabela 2: Proposta de interpretação dos teores de P do solo extraído por Mehlich 3 para os quatro grupos de culturas sugeridos pela CQFS - RS/SC (2016) e conforme os teores de argila

\begin{tabular}{|c|c|c|c|c|}
\hline \multirow{3}{*}{ Interpretação } & \multicolumn{4}{|c|}{ Teor de Argila (\%) } \\
\hline & $>60$ & $41-60$ & $21-40$ & $<20$ \\
\hline & \multicolumn{4}{|c|}{ Grupo $1, \mathrm{mg} \mathrm{dm}^{-3}$} \\
\hline Muito baixo & $\leq 4,0$ & $\leq 6,0$ & $\leq 11,0$ & $\leq 22,0$ \\
\hline Baixo & $4,1-8,0$ & $6,1-12,0$ & $11,1-22,0$ & $22,1-44,0$ \\
\hline Médio & $8,1-12,0$ & $12,1-18,0$ & $22,1-33,0$ & $44,1-66,0$ \\
\hline Alto & $12,1-24,0$ & $18,1-36,0$ & $33,1-66,0$ & $66,1-132,0$ \\
\hline \multirow[t]{2}{*}{ Muito alto } & $>24,0$ & $>36,0$ & $>66,0$ & $>132,0$ \\
\hline & \multicolumn{4}{|c|}{ Grupo $2, \mathrm{mg} \mathrm{dm}^{-3}$} \\
\hline Muito baixo & $\leq 2,5$ & $\leq 3,5$ & $\leq 7$ & $\leq 13,0$ \\
\hline Baixo & $2,6-5,0$ & $3,6-7,0$ & $7,1-14,0$ & $13,1-26,0$ \\
\hline Médio & $5,1-7,5$ & $7,1-10,5$ & $14,1-21,0$ & $26,1-39,0$ \\
\hline Alto & $7,6-15,0$ & $10,6-21,0$ & $21,1-42,0$ & $39,1-78,0$ \\
\hline \multirow[t]{2}{*}{ Muito alto } & $>15,0$ & $>21,0$ & $>42,0$ & $>78,0$ \\
\hline & \multicolumn{4}{|c|}{ Grupo $3, \mathrm{mg} \mathrm{dm}^{-3}$} \\
\hline Muito baixo & $\leq 1,0$ & $\leq 1,5$ & $\leq 4,0$ & $\leq 6,5$ \\
\hline Baixo & $2,1-2,0$ & $1,6-3,0$ & $4,1-8,0$ & $6,6-13,0$ \\
\hline Médio & $2,1-3,0$ & $3,1-4,5$ & $8,1-12,0$ & $13,1-19,5$ \\
\hline Alto & $3,1-6,0$ & $4,6-9,0$ & $12,1-24,0$ & $19,6-39,0$ \\
\hline \multirow[t]{2}{*}{ Muito alto } & $>6,0$ & $>9,0$ & $>24,0$ & $>39,0$ \\
\hline & \multicolumn{4}{|c|}{ Grupo $4, \mathrm{mg} \mathrm{dm}^{-3}$} \\
\hline Muito baixo & & $\leq 2,3$ & & \\
\hline Baixo & & $2,3-4,6$ & & \\
\hline Médio & & $4,7-6,9$ & & \\
\hline Alto & & $7,0-13,8$ & & \\
\hline Muito alto & & $>13,8$ & & \\
\hline
\end{tabular}

Rev. Ceres, Viçosa, v. 65, n.6, p. 546-554, nov/dez, 2018 
de 21 a $40 \%:$ P-M1 $=$ P-M3*1,0; solos com argila de $>$ 40\%: $\mathrm{P}-\mathrm{M} 1_{\text {eq }}=\mathrm{P}-\mathrm{M} 3 * 0,80$.

A partir dos resultados obtidos, sugere-se ainda a utilização de tabelas de interpretação da disponibilidade de P para os valores extraídos com M-3 (Tabela 2). Ressalta-se que os valores apresentados foram arredondados para números inteiros, prática que é realizada para fins de praticidade nos manuais de adubação. A tabela é baseada no manual da CQFS - RS/SC (2016), sendo os valores derivados das equações de correlação linear geradas na comparação entre os valores de P extraídos por M-1 e M-3. Além disso, a tabela apresenta as classes de $\mathrm{P}$ para os diferentes grupos de culturas segundo sua resposta ao P. A separação das culturas em grupos de exigência em $\mathrm{P}$ foi introduzida no novo manual de calagem e adubação da CQFS - RS/SC (2016), objetivando separar culturas como "pouco exigentes", "exigentes" ou "muito exigentes" em P. Para o grupo das "pouco exigentes", os níveis críticos correspondem a 50\% daqueles para as culturas “exigentes”. Já para o grupo das culturas "muito exigentes", os níveis críticos correspondem a $170 \%$ daqueles para as culturas "exigentes". Existe ainda um quarto grupo em separado, representado apenas pela cultura do arroz irrigado em razão da dinâmica e disponibilidade de $\mathrm{P}$ no solo ser completamente diferente em solos anoxicos.

Os resultados encontrados neste estudo demonstram que ambos os métodos de extração podem ser utilizados em laboratórios de rotina para estimar a disponibilidade de $\mathrm{P}$ em solos catarinenses. Deve ser dada preferência (exceto para solos adubados recentemente com fosfatos naturais) ao método M-1, por apresentar extensa calibração de campo para os solos de SC e RS realizada nos últimos 50 anos. Para solos que tenham recebido adubação com fosfatos naturais a menos de dois anos da análise, o extrator M-1 não é indicado (CQFS - RS/ SC, 2016), recomendando-se a extração pelo método M3 (CQFS - RS/SC, 2016). O método da RTA também pode ser utilizado para solos fertilizados com fosfatos naturais, apresentando boa correlação com M-1. Destaca-se, contudo, que ambos os métodos testados sofrem interferência do conteúdo de argila do solo, a qual deve ser considerada para correta determinação das classes de disponibilidade de $\mathrm{P}$ e posterior recomendação de adubação. Além disso, a utilização de uma única equação para converter os valores lidos por M-3 para um valor de M-1 equivalente pode apresentar grande variabilidade, ressaltando-se a necessidade de investimento de esforços de pesquisa para a calibração direta do M-3 à campo, relacionando os teores extraídos pelo método e a absorção de $\mathrm{P}$ e produtividade das plantas, da mesma maneira como foi feita para o M-1 durante a extensa calibração realizada para M-1 no Sul do Brasil.

\section{CONCLUSÕES}

Os métodos Mehlich-3 e resina extraem, em média, 11 a 12\% mais fósforo que o Mehlich-1, mas apresentam elevada correlação com o método padrão, podendo ser utilizados como substituintes do mesmo.

Os métodos Mehlich-1, Mehlich-3 e resina foram sensíveis ao teor de argila do solo, extraindo quantidades menores de fósforo em solos mais argilosos.

O método Mehlich-3 extrai mais fósforo que o Mehlich1 em solos arenosos e menor quantidade do nutriente que o Mehlich-1 em solos argilosos, podendo-se usar a equação $\left[\mathrm{P}-\mathrm{M} 1_{\text {eq }}=(\mathrm{P}-\mathrm{M} 3 /(1,38-(0,01 *\right.$ *arg $)]$ para transformar os teores de fósforo extraídos por Mehlich-3 em valores equivalentes a Mehlich-1 para a interpretação da disponibilidade de $\mathrm{P}$ para as plantas.

\section{REFERÊNCIAS}

Almeida JA, Ernani PR \& Maçaneiro KC (1999) Recomendação alternativa de calcário para solos altamente tamponados do Extremo Sul do Brasil. Ciência Rural, 29:651-656.

Bortolon L, Gianello C \& Schlindwein JA (2009) Avaliação da disponibilidade de fósforo no solo para o milho pelos métodos Mehlich-1 e Mehlich-3. Scientia Agraria, 10:05-312.

Bortolon L, Gianello C, Welter S, Almeida RGO \& Giasson E (2011) Simultaneous extraction of phosphorus, potassium, calcium and magnesium from soils and potassium recommendations for crops in Southern Brazil. Pedosphere, 21:365-372.

Bortolon L \& Gianello C (2008) Interpretação de resultados analíticos de fósforo pelos extratores Mehlich-1 e Mehlich-3 em solos do Rio Grande do Sul. Revista Brasileira de Ciência do Solo, 32:2751-2756.

Bortolon L \& Gianello C (2012) Multielement extraction from Southern Brazilian soils. Communications in Soil Science and Plant Analysis, 43:1615-1624.

Camargo LA, Marques Júnior J, Pereira GT \& Alleoni LRF (2013) Spatial correlation between the composition of the clay fraction and contents of available phosphorus of an Oxisol at hillslope scale. Catena, 100:100-106.

Chien SH, Edmeades D, Mcbride R \& Sahrawat KL (2014) Review of maleic-itaconic acid copolymer purported as urease inhibitor and phosphorus enhancer in soils. Agronomy Journal, 106:423-430.

CQFS - Comissão de Química e Fertilidade do Solo (2016) Manual de calagem e adubação para os Estados de Rio Grande do Sul e de Santa Catarina. Viçosa, Sociedade Brasileira de Ciência do Solo. 376p.

Embrapa - Empresa Brasileira de Pesquisa Agropecuária (1997) Manual de métodos de análise de solos. Rio de Janeiro, Embrapa. 225p.

Eriksson AK, Gustafsson JP \& Hesterberg D (2015) Phosphorus speciation of clay fractions from long-term fertility experiments in Sweden. Geoderma, 241-242:68-74.

Ferreira DF (2014) Sisvar: a Guide for its Bootstrap procedures in multiple comparisons. Ciência e Agrotecnologia, 38:109-112.

Fink JR, Inda AV, Bavaresco J, Barrón V, Torrent J \& Bayer C (2016) Adsorption and desorption of phosphorus in subtropical soils as affected by management system and mineralogy. Soil \& Tillage Research, 155:62-68. 
Freitas IF, Novais RF, Villani EMA \& Novais SV (2013) Phosphorus extracted by ion exchange resins and Mehlich-1 from Oxisols (Latosols) treated with different phosphorus rates and sources for varied soil-source contact periods. Revista Brasileira Ciência do Solo, 37:667-677.

Gatiboni LC, Brunetto G, Kaminski J, Rheinheimer DS, Ceretta CA \& Basso CJ (2008) Soil phosphorus forms after successive pig slurry application in a native pasture. Revista Brasileira de Ciência do Solo, 32:1753-1761.

Gatiboni LC, Kaminski J \& Rheinheimer DS (2005) Modificações nas formas de fósforo do solo após extrações sucessivas com Mehlich-1, Mehlich-3 e Resina Trocadora de Ânions. Revista Brasileira de Ciência do Solo, 29:363:371.

Mehlich A (1978) New extractant for soil test evaluation of phosphorus, potassium, magnesium, calcium, sodium, manganese and zinc. Communications in Soil Science and Plant Analysis, 9:477-492

Mehlich A (1984) Mehlich 3 soil test extractant: A modification of Mehlich 2 extractant. Communications in Soil Science and Plant Analysis, 15:1409-1416.

Miola GR (1995) Extração de P, K, Ca e Mg do solo por diferentes métodos e avaliação da disponibilidade de fósforo para as plantas. Dissertação de Mestrado. Universidade Federal do Rio Grande do Sul, Porto Alegre. 140p.

Murphy J \& Riley JP (1962) A modified single solution method for the determination of phosphate in natural waters. Analytica Chimica Acta, 27:31-36

Oliveira CMB, Gatiboni LC, Ernani PR, Boitt G \& Brunetto G (2015) Capacidade de predição da disponibilidade de fósforo em solo com aplicação de fosfato solúvel e natural. Científica, 43:413-419.
Oliveira CMB (2010) Comportamento dos métodos Mehlich 1, Mehlich 3 e resina trocadora de ânions frente ao poder tampão do solo e à utilização de fosfatos naturais. Dissertação de Mestrado. Universidade do Estado de Santa Catarina, Lages. $71 \mathrm{p}$.

Oliveira Júnior AO, Prochnow LI \& Klepker D (2011) Soybean yield in response to application of phosphate rock associated with triple superphosphate. Scientia Agrícola, 68:376-385.

Santos DR, Gatiboni LC \& Kaminski J (2008) Fatores que afetam a disponibilidade do fósforo e o manejo da adubação fosfatada em solos sob sistema plantio direto. Ciência Rural, 38:576:586.

Schlindwein JA, Bortolon L \& Gianello C (2011) Soil phosphorus available for crops and grasses extracted with three soil-test methods in Southern Brazilian soils amended with phosphate rock. Communications in Soil Science and Plant Analysis, 42:283-292.

Schlindwein JA \& Gianello C (2008) Nível de suficiência e índice de equivalência entre o fósforo determinado pelos métodos da resina de troca iônica e Mehlich-1. Revista Brasileira de Agrociência, 14:299-306.

Steffens D, Leppin T, Ebengreuth NL, Yang ZM \& Schubert S (2010) Organic soil phosphorus considerably contributes to plant nutrition but is neglected by routine soil-testing methods. Journal of Plant Nutrition and Soil Science, 173:765-771.

Steiner F, Lana MC, Zoz T, Frandoloso JF \& Fey R (2012) Extraction methods and availability of phosphorus for soybean in soils from Paraná State, Brazil. Semina: Ciências Agrárias, 33:1005-1014.

Tedesco HJ, Volkweiss SJ \& Bohnen H (1995) Análises de solo, plantas e outros materiais. Porto Alegre, Universidade Federal do Rio Grande do Sul. 50p. (Boletim técnico, 5). 